${ }^{8} \mathrm{M}$ Gatzoulis, ${ }^{1} \mathrm{~A}$ J Peacock. ${ }^{1}$ Scottish Pulmonary Vascular Unit, Glasgow, UK; ${ }^{2}$ Pulmonary Vascular Unit, Royal Hallamshire Hospital, Sheffield, UK; ${ }^{3}$ Pulmonary Hypertension Service, Hammersmith Hospital, London, UK; ${ }^{4}$ Pulmonary Vascular Disease Unit, Papworth Hospital, Cambridge, UK; ${ }^{5}$ Northern Pulmonary Vascular Unit, Freeman Hospital, Newcastle, UK; ${ }^{6}$ Pulmonary Hypertension Unit, Mater Misericordiae Hospital, Dublin, Ireland; ${ }^{7}$ Pulmonary Hypertension Unit, Royal Free Hospital, London, UK; ${ }^{8}$ Royal Brompton Pulmonary Hypertension and Adult Congenital Heart Centre, Royal Brompton Hospital, London, UK

Introduction and Objectives The age of patients with idiopathic pulmonary arterial hypertension (IPAH) has increased since the $\mathrm{NIH}$ registry. It is postulated that older IPAH patients may have a different disease phenotype compared to their younger counterparts.

Methods Retrospective observational study of all consecutive incident cases of IPAH, heritable and anorexigen-associated pulmonary arterial hypertension diagnosed in all eight pulmonary hypertension centres in the UK and Ireland between 1st January 2001 and 31st December 2009. Patients were divided into younger and older subgroups by the median age ( 57 years).

Results Of 646 incident cases (mean age $=54$ ), 22\% were over the age of 70 and $3 \%$ over age 80 . Younger patients had higher $\%$ female ( $71 \%$ vs $56 \%$ ), shorter duration of symptoms (median 15 months vs 21 months), better functional class (18\% in functional class I/II vs $10 \%$ ), exercise capacity (6-minute walk distance $325 \mathrm{~m}$ vs $217 \mathrm{~m}$ ) and higher \% predicted DLCO (65\% vs $47 \%$ ) compared to older patients. Older patients were more likely to present with peripheral oedema ( $41 \%$ vs $28 \%$ ) whereas younger patients were more likely to complain of syncope (30\% vs $10 \%)$, presyncope (14\% vs $7 \%$ ) and fatigue $(15 \%$ vs $9 \%)$ at the time of diagnosis. Younger patients had higher mean pulmonary artery pressure $(56 \mathrm{~mm} \mathrm{Hg}$ vs $49 \mathrm{~mm} \mathrm{Hg}$ ) and pulmonary vascular resistance index (24 WU.m ${ }^{2}$ vs 21 WU. $\left.{ }^{2}\right)$ but lower wedge pressure ( $9 \mathrm{~mm} \mathrm{Hg}$ vs $10 \mathrm{~mm} \mathrm{Hg}$ ) compared to older patients. Patients in the highest age quartile had the worst survival [Abstract S71 figure 1].

Conclusion Older incident IPAH patients appear to have a different disease phenotype compared to younger patients. IPAH is no longer a disease that affect predominantly young female only.

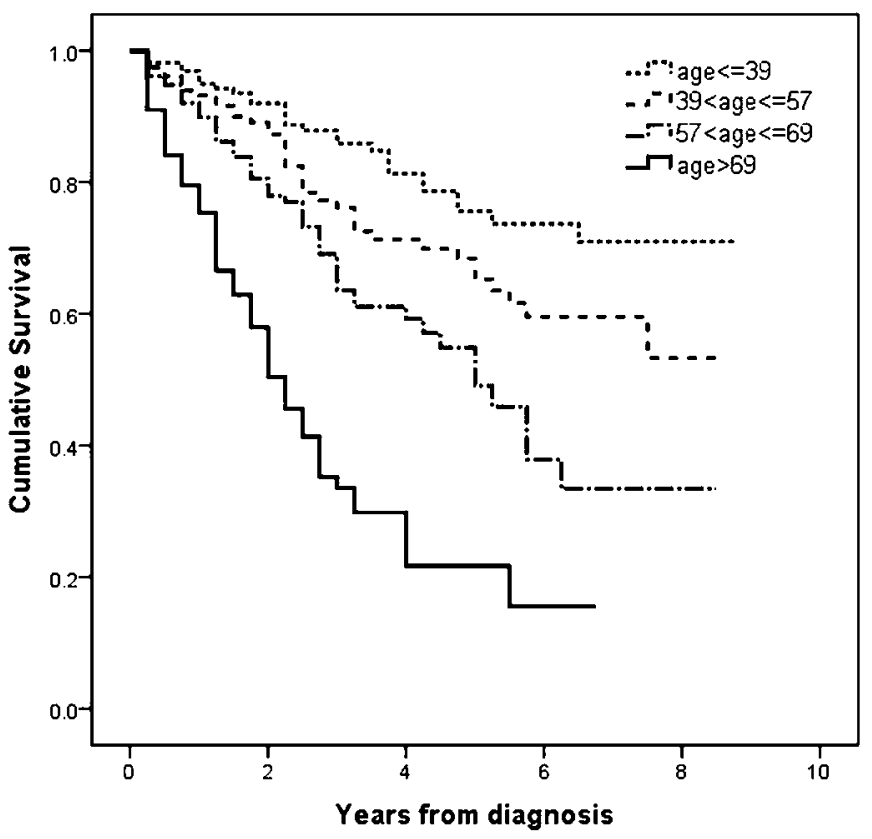

Abstract S71 Figure 1 Survival of incident idiopathic, heritable and anorexigen-associated pulmonary arterial hypertension by age quartiles.

\section{S72 PREDICTION OF SURVIVAL IN PULMONARY ARTERIAL HYPERTENSION USING SURVIVAL EQUATIONS. RESULTS FROM THE PULMONARY HYPERTENSION REGISTRY OF THE UK AND IRELAND}

doi:10.1136/thoraxjnl-2011-201054b.72

${ }^{1} Y$ Ling, ${ }^{1} \mathrm{M}$ K Johnson, ${ }^{2} \mathrm{D}$ Kiely, ${ }^{2} \mathrm{R}$ Condliffe, ${ }^{2} \mathrm{C}$ Elliot, ${ }^{3} \mathrm{~S}$ Gibbs, ${ }^{3} \mathrm{~L}$ Howard, ${ }^{4} \mathrm{~J}$ PepkeZaba, ${ }^{4} \mathrm{~K}$ Sheares, ${ }^{5} \mathrm{P}$ Corris, ${ }^{5} \mathrm{~A}$ Fisher, ${ }^{5} \mathrm{~J}$ Lordan, ${ }^{6} \mathrm{~S}$ Gaine, ${ }^{7} \mathrm{G}$ Coghlan, ${ }^{8} \mathrm{~J}$ Wort, ${ }^{8} \mathrm{M}$ Gatzoulis, ${ }^{1} \mathrm{~A} J$ Peacock. ${ }^{1}$ Scottish Pulmonary Vascular Unit, Golden Jubilee National Hospital, Glasgow, UK; ${ }^{2}$ Pulmonary Vascular Unit, Royal Hallamshire Hospital, Sheffield, UK; ${ }^{3}$ Pulmonary Hypertension Service, Hammersmith Hospital, London, UK; ${ }^{4}$ Pulmonary Vascular Disease Unit, Papworth Hospital, Cambridge, UK; ${ }^{5}$ Northern Pulmonary Vascular Unit, Freeman Hospital, Newcastle, UK; ${ }^{6}$ Pulmonary Hypertension Unit, Mater Misericordiae Hospital, Dublin, Ireland; ${ }^{7}$ Pulmonary Hypertension Unit, Royal Free Hospital, London, UK; ${ }^{8}$ Royal Brompton Pulmonary Hypertension and Adult Congenital Heart Centre, Royal Brompton Hospital, London, UK

Introduction The NIH survival equation ${ }^{1}$ was developed in the 1980 s and used in many clinical trials to show survival benefit. Since then, significant advances had been made in the diagnosis and management of pulmonary arterial hypertension (PAH). Several new equations have been developed to better predict survival in $\mathrm{PAH}$. All these survival equations were derived using prognostic factors identified from a mixed incident and prevalent PAH cohort. However, prevalent patients have better survival compared to incident patients.

Objectives To determine applicability of currently available survival equations in incident idiopathic (IPAH), heritable and anorexigenassociated PAH.

Methods Retrospective observational study of all incident cases of IPAH, heritable and anorexigen-associated PAH diagnosed in the UK and Ireland between 1st January 2001 and 31st December 2009. Observed survival in the UK and Ireland was estimated using life table analysis and compared with predicted survival in the same patients calculated using the NIH, ${ }^{1}$ French, ${ }^{2}$ Pulmonary Hypertension Connection $(\mathrm{PHC})^{3}$ and $\mathrm{REVEAL}^{4}$ Registry equations. Results Total of 646 patients were diagnosed over the study period. Observed vs predicted survival were compared [Abstract S72 figure 1].

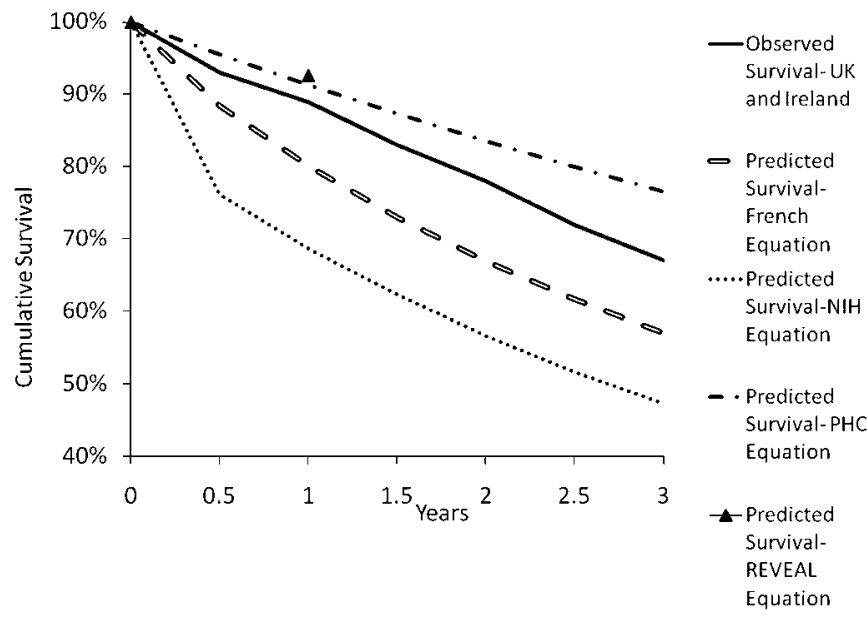

Abstract S72 Figure 1 Observed vs predicted survival using the $\mathrm{NIH}$, French, PHC and REVEAL equations.

Conclusion Survival equations derived from other mixed incident and prevalent pulmonary hypertension populations may not accurately predict survival of incident pulmonary arterial hypertension from the UK and Ireland. Differences in baseline characteristics, treatment practice and time period between our patients and survival equations derivation populations need to be taken into account when applying these equations in daily clinical practice. 


\section{REFERENCES}

D'Alonzo, et al. Ann Int Med 1991

Humbert, et al. ERJ 2010

Thenappan, et al. ERJ 2010.

4. Benza, et al. Circulation 2010.

\section{Cell pathways in lung inflammation and injury S73 MACROPHAGES AS VEHICLES FOR DELIVERING CELL THERAPY TO INJURED LUNG}

doi:10.1136/thoraxjnl-2011-201054b.73

K McNulty, C J Scotton, E K Sage, R C Chambers, S M Janes. University College London, London, UK

Introduction Injury to the alveolar epithelium underlies a number of important lung diseases, exemplified by the syndromes of acute lung injury and acute respiratory distress syndrome, which currently have a poor prognosis. Keratinocyte growth factor (KGF) is a mitogen for, and exerts beneficial effects on, type II alveolar epithelial cells. Pre-treatment with KGF is associated with improvements in outcomes in animal models of lung injury, but the use of recombinant KGF as a clinical therapy is limited by its short bioavailability and lack of specificity. We sought to determine whether macrophages could be used as vehicles to deliver KGF therapy to the injured lung.

Methods Macrophages from a murine macrophage cell line (IC-21) were transduced with a lentiviral vector expressing KGF and the reporter gene GFP. Mice were given oropharyngeal (OP) bleomycin to induce lung injury. On days one and three after induction of lung injury, mice were given $3 \times 10^{6}$ KGF-transduced IC-21 cells (or controls) by $\mathrm{OP}$ instillation. Mice were sacrificed on day 5 , and bronchoalveolar lavage fluid (BALF) was harvested and lungs were processed for histology. For in vivo tracking experiments, IC-21 macrophages were transduced with a lentiviral vector expressing luciferase and mice were imaged longitudinally using real-time bioluminescence imaging.

Results KGF-expression was confirmed in KGF-lentivirus-transduced macrophages, however delivery of these cells was not associated with improvements in measures of alveolar-capillary membrane permeability (BALF albumin) or inflammation (total and differential cell counts) after lung injury. Cells expressing GFP were recovered in BALF, and immunohistochemistry showed groups of cells close to conducting airways. Longitudinal imaging of mice after OP delivery of luciferase-transduced IC-21 cells suggested that cells initially localised to the lungs, but did not persist at $48 \mathrm{~h}$ after delivery.

Conclusions KGF-expressing macrophages can be generated using lentiviral vectors, but therapeutic delivery of these cells to the lungs did not improve measured outcomes in the mouse bleomycin lung injury model. Longitudinal imaging suggested that the lack of therapeutic efficacy of KGF-transduced macrophages may be due to their limited survival, and future work should focus on optimising macrophage delivery and survival in vivo.

\section{S74 INCREASED PLASMA LEVELS OF SYNDECAN-1 AND sFLT-1 DURING CARDIOPULMONARY BYPASS SURGERY: ASSOCIATIONS WITH SRAGE}

doi:10.1136/thoraxjnl-2011-201054b.74

J Zhang, B C Creagh-Brown, S Mumby, M J Griffiths, A Burke-Gaffney. Unit of Critical Care, NHLI, Imperial College London, London, UK

Introduction and Objectives Endothelial barrier dysfunction contributes to the systemic inflammatory response syndrome (SIRS) for which cardiac surgery necessitating cardiopulmonary bypass $(\mathrm{snCPB})$ is a human model. A growing body of evidence suggests that the receptor for advanced glycation end products (RAGE) is also implicated in the pathogenesis of SIRS. While RAGE activation, in vitro, is known to decrease barrier function of cultured endothelial cells, the role of RAGE in regulating vascular permeability in patients undergoing snCPB is unknown. We hypothesised that plasma levels of markers of endothelial barrier dysfunction, syndecan-1, an endothelial glycocalyx component; and sFlt-1, a soluble form of the vascular endothelial cell growth factor (VEGF) receptor-1, will increase during $\mathrm{snCPB}$ and levels relate to patient outcome.

Methods ELISA measurements/western blotting was used to determine syndecan-1 and sFlt-1 levels in pre-, intra- and post-snCPB plasma samples; for comparison, glypican-1, another glycocalyx component and Robo4, an endothelial barrier stabilising protein, were also measured. Clinical indices: age, nature of operation, CPB time, ischaemic time, intensive care unit (ICU)/ hospital length of stay (LOS) were collected from electronic databases.

Results Syndecan-1 was significantly higher during CPB $(77.17 \pm 39.72 \mathrm{ng} / \mathrm{ml}) \quad$ compared with pre-snCPB levels $(35.26 \pm 25.81 \mathrm{ng} / \mathrm{ml}, \mathrm{n}=14, \mathrm{p}<0.01)$. Plasma levels of $\mathrm{sFLt}-1$ were significantly $(\mathrm{p}<0.001)$ higher during $(10000 \mathrm{pg} / \mathrm{ml} \pm 3601, \mathrm{n}=10)$ and post-snCPB $(4282 \pm 3271 \mathrm{pg} / \mathrm{ml})$ compared with preoperative levels $(69.68 \pm 35.22 \mathrm{pg} / \mathrm{ml})$. Preoperative plasma syndecan- 1 correlated positively with ICU LOS $\left(r^{2}=0.486, p=0.006\right)$; whereas, intraoperative sFlt- 1 correlated negatively with ICU LOS $\left(r^{2}=0.406\right.$, $\mathrm{p}=0.048$ ). Intraoperative syndecan- 1 levels positively associated with ischaemic time $\left(r^{2}=0.383, p=0.018\right)$ and plasma sRAGE levels $\left(r^{2}=389, p=0.040\right)$; postoperative syndecan-1 levels correlated with sRAGE $\left(r^{2}=0.790, p=0.0003\right)$. Glypican-1 and Robo4 were also detected in snCPB plasma samples.

Conclusion Plasma levels of syndecan-1 and sFlt-1 were highest during snCPB. A positive association between preoperative syndecan-1 and ICU LOS is consistent with a relationship between endothelial barrier dysfunction and outcome. By contrast, higher intraoperative sFlt-1 correlating with shorter ICU LOS implied a protective role of sFlt-1. Associations between syndecan-1 and sRAGE suggest a link between RAGE and endothelial barrier dysfunction that merits further investigation; as do the novel findings that glypican-1 and Robo4 were detected in plasma of patients undergoing snCPB.

\section{S75 DO “CLINICALLY RELEVANT" TIDAL VOLUMES REALLY CAUSE VENTILATOR-INDUCED LUNG INJURY IN MICE?}

doi:10.1136/thoraxjnl-2011-201054b.75

M R Wilson, B V Patel, M Takata. Imperial College London, London, UK

Background Ventilator-induced lung injury (VILI) caused by excessive lung stretch during mechanical ventilation, is an important determinant of intensive care mortality. In recent years the mouse has increasingly become the pre-clinical model of choice, and studies using mice have identified numerous pathways and mediators all apparently vital during VILI. However, findings have not translated into clinical benefit, and it is conceptually extremely difficult to reconcile this plethora of mediators into a single paradigm. We propose that this confused situation has arisen from a somewhat naive belief that the wide variety of tidal volumes $\left(V_{T}\right)$ used within such studies all induce over-stretching of the lungs.

Methods Anaesthetised mice were ventilated $\left(3 \mathrm{~cm} \mathrm{H}_{2} \mathrm{O}\right.$ positive end-expiratory pressure, using air $\pm \mathrm{CO}_{2}$ to regulate $\mathrm{pH}$ ) with a variety of $\mathrm{V}_{\mathrm{T}}$ ranging from "clinically relevant" $(10 \mathrm{ml} / \mathrm{kg})$ to "very high" $(40 \mathrm{ml} / \mathrm{kg})$ for up to $3 \mathrm{~h}$.

Results Both $10 \mathrm{ml} / \mathrm{kg}$ and $40 \mathrm{ml} / \mathrm{kg} \mathrm{V}_{\mathrm{T}}$ evoked deterioration in arterial $\mathrm{pO}_{2}$ and mean arterial blood pressure (BP), although intermediate $V_{\mathrm{T}}(20-30 \mathrm{ml} / \mathrm{kg})$ did not (see Abstract $\mathrm{S} 75$ table 1$)$. Lung 